\title{
Antidepressant Medication Use during Breastfeeding
}

\author{
Teresa Lanza di Scalea, M.D. and \\ Department of Psychiatry, Western Psychiatric Institute and Clinic University of Pittsburgh, Medical \\ Center 808 Bellefield Towers; 15213 Pittsburgh (PA); Tel: 412246 6974; \\ lanzadiscaleat@upmc.edu, Department of Neurosciences, Operative Unit of Psychiatry, University \\ of Rome Tor Vergata Via Nomentana 1362, 00100 Roma; Tel: 0641400129 ; Fax: 0641400343

\section{Katherine L. Wisner, M.D.} \\ Professor of Psychiatry, Obstetrics and Gynecology and Reproductive Sciences, Epidemiology, and \\ Women's Studies, Women's Behavioral HealthCARE, Western Psychiatric Institute and Clinic, \\ University of Pittsburgh Medical Center, 3811 O'Hara Street, Pittsburgh, PA 15213, Tel: 412246 \\ 6564; Fax: 412246 6069, WisnerKL@upmc.edu
}

\begin{abstract}
We performed an electronic search by using MEDLINE, PreMEDLINE, Current Contents, Biological Abstracts, and PsycINFO from June 2002 through December 2008 using the following terms: "antidepressant drugs", "antidepressive agents", "human milk", "lactation", and "breastfeeding" and the generic name of each antidepressant. Articles in the English language with reports of antidepressants in maternal serum or breast milk, infant serum, and short-term and longterm clinical outcomes in the infants were obtained. The search yielded a total of 31 empirical papers. Breastfeeding and antidepressant treatments are not mutually exclusive. Sertraline, paroxetine, nortriptyline and imipramine are the most evidence-based medications for use during breastfeeding.
\end{abstract}

\section{Keywords}

Antidepressant; breastfeeding; nursing infant

\begin{abstract}
Human milk represents the ideal primary source of nutrients, immunological defenses, and growth-promoting factors for the term and preterm newborn, and provides the mother-infant dyad with major short and long-term health benefits. Breastfed infants have less risk of mortality and morbidity (i.e. gastrointestinal and respiratory infections, urinary infections, sepsis, meningitis, necrotizing enterocolitis). Breastfeeding continues to offer health benefits into and after early childhood. These include decreased risk for asthma, inflammatory bowel disease, diabetes mellitus and hematological cancers during infancy, as well as lower rate of obesity and asthma in adolescence. Breastfeeding also provides benefits for the mother's health. It has been associated with reduced risk of ovarian cancer and breast cancer, as well as post partum weight retention and bleeding (1)(2). Moreover, the intimate bodily contact during breastfeeding comprises the initial interpersonal communication and facilitates mother -infant attachment. Both the American Academy of Pediatrics and the World Health Organization recommended the use of exclusive breast milk for 6 months for all infants with the option of adequate substitutes only for infants who cannot be breast fed (2)(3).
\end{abstract}

CORRESPONDING AUTHOR: Katherine L. Wisner, M.D., Women's Behavioral HealthCARE, Western Psychiatric Institute and Clinic University of Pittsburgh Medical Center, 3811 O`Hara Street, Pittsburgh, PA 15213, Tel: 4122466564 Fax: 412246 6069,

WisnerKL@upmc.edu.

Dr. Lanza di Scalea reports no competing interests. 
The prevalence of postpartum depression (PPD) has been estimated as high as $14.5 \%$ (4). Depressed mothers may prefer to avoid pharmacological treatment because of concerns about adverse effects in the nursing infants (5)(6). Women with postpartum depression who are breastfeeding receive fewer prescriptions for psychotropic medications compared to nonbreastfeeding women and are more likely to choose a non-pharmacological treatment (5). Although psychotherapy has been demonstrated to be efficacious in treating PPD (6), it is not widely available in public settings. The high prevalence of untreated PPD is a major health issue due to its association with functional impairment in the mother, elevated risk of psychopathology in the children as well as impacts on the mother-infant relationship (7)(8).

Considering the use of antidepressants during lactation is a common clinical conundrum due to the frequency with which PPD occurs. Antidepressant medications are also appropriate for the treatment of other disorders common in women of childbearing age, such as panic disorder, generalized anxiety disorder, obsessive-compulsive disorder and bulimia (9). Therefore, physicians will frequently encounter challenging questions such as: 1) What are the risks of untreated maternal illness? 2) Are breastfeeding and antidepressant drug treatment mutually exclusive? 3) Is there evidence to support that specific antidepressants are more favorable for use during breastfeeding? 4) What is known about the more recently released antidepressants? 5) Are there practical strategies that reduce the infant's exposure to antidepressants ? 6) How can potential adverse effects related to drug transmission through breast milk be monitored in the infant?

The available evidence on the effects of antidepressants during lactation largely consists of case reports and studies of small samples. A detailed review and pooled analysis of antidepressant levels in lactating mothers, breast milk and nursing infants was published in the American Journal of Psychiatry in June, 2004 (10). This paper included publications through June, 2002, from searches of MEDLINE, PreMEDLINE, Current Contants, Biological Abstracts and PsychINFO (through July 2002) plus bibliographic searches of these identified articles. Results of the pooled analyses indicated that breastfed infants exposed to paroxetine and sertraline were unlikely to develop detectable serum drug levels. Infants exposed to fluoxetine through breast milk were more likely to develop elevated levels of the drug especially if the mothers started the treatment during pregnancy (due to prenatal loading). The data on citalopram were limited compared the other SSRIs, but suggested that some infants developed quantifiable serum levels of the drug which may be associated with adverse effects.

Weissman et al. (10) reported that short-term adverse effects had been identified for doxepin (a tricyclic), citalopram, paroxetine and fluoxetine, whereas data on the long-term effects were limited. The authors concluded that breastfeeding women may reasonably choose to use antidepressants and that routine laboratory measurement of infant serum drug levels for clinical purposes was not warranted. The authors suggested the evaluation of serum infant levels as a direct measure of infant exposure in future research, since infant exposure to drugs is influenced not only by the amount ingested through breastfeeding but also by capacity to metabolize the drug.

The goals of this paper are to: 1) Provide the results of a literature search with the same databases as in Weissman et al.(10) from the end date of their search (June, 2002) through December, 2008. 2) Compare the data derived from the newer set of papers to the original publications to determine if a change in recommendations is appropriate. 3) Present data from recently released antidepressants and provide clinical guidance on their use. 


\section{METHODS}

An electronic search was performed by using MEDLINE, PreMEDLINE, Current Contents, Biological Abstracts, and PsycINFO from June 2002 through December 2008 using the following key search terms: "antidepressant drugs", "antidepressive agents", "human milk", "lactation", and "breastfeeding" and the generic name of each antidepressant agent. Articles in the English language with reports of antidepressants in maternal serum or breast milk, infant serum, and short-term and long-term clinical outcomes in the infants were obtained. This procedure yielded a total of 63 papers reporting the following types of publications: editorials $(\mathrm{N}=2)$, letters to the editor $(\mathrm{N}=5)$; reviews with practical recommendations by the authors $(\mathrm{N}=25)$; practical guidelines developed by scientific commissions $(\mathrm{N}=3)$; empirical papers $(\mathrm{N}=31)$, of which 12 were single case reports and 19 were small samples case series $(\mathrm{N} \leq 20)$. We identified empirical papers listed as follows: serotonergic selective reuptake (SSRIs) $(\mathrm{N}=20)$; serotonin-noradrenergic reuptake inhibitors (SNRIs)(N=4), respectively venlafaxine $(\mathrm{N}=3)$ and duloxetine $(\mathrm{N}=1)$; tricyclic/heterocyclic antidepressants (TCAs) $(\mathrm{N}=1)$; noradrenergic and specific serotonergic antidepressant (NaSSA: mirtazapina) $(\mathrm{N}=3)$; and norepinephrine dopamine reuptake inhibitor (NDRI: bupropion) $(\mathrm{N}=5)$.

\section{TRICYCLIC/HETEROCICLYC ANTIDEPRESSANTS (TCA)}

The tricyclic and heterocyclic antidepressants exhibit their therapeutic effect through blocking reuptake of norepinephrine, dopamine and serotonin, with the level of blockade dependent upon the specific agent. Tricyclic antidepressants are associated with cardiac toxicity in overdose as well as a variety of adverse effects due to their multiple receptor impacts. As a consequence, the use of tricyclics has decreased in clinical practice. However, a double blind randomizated clinical trial of nortriptyline (TCA) vs. sertraline (SSRI) for PPD identified similar rates of both response and remission (11). The rate of side effects or impairment due to side effects did not differ in the nortriptyline compared to the sertraline treated women. Although the side effect burden did not differ in the TCA or SSRI-treated groups, the specific profiles did, with dry mouth, thirst, constipation in the TCA-treated group and headaches, perspiration, hot flashes in the SSRI-treated group (11). Breastfeeding women were included in this randomized clinical trial. Sixteen women in the NTP group were breastfeeding and provided mother-infant samples. The mean age of the nursing infants was 5.9 weeks. Infant NTP serum levels ranged from non-detectable (ND) to $4 \mathrm{ng} / \mathrm{ml}$; E-10-hyroxyNTP ranged from $\mathrm{ND}$ to $7 \mathrm{ng} / \mathrm{ml}$, and Z-10-hydroxyNTP ranged from ND to $8 \mathrm{ng} / \mathrm{ml}$. No adverse events were noted in the infants.

The majority of the available infant serum level data for TCAs includes NTP and imipramine, which in most cases are not detectable and have not been associated with adverse clinical events. Doxepin (which is very sedating and has a metabolite with a long half-life) has been associated with sedation and respiratory depression and has been widely considered contraindicated (12)(13). TCAs can be considered first choice treatments for women with PPD when the mother has been treated successfully for past episodes and there are no current contraindications for their use, such as suicidality.

\section{SELECTIVE SEROTONIN-REUPTAKE INHIBITORS (SSRI)}

Beyond the primary activity of selective inhibition of serotonergic reuptake, different SSRIs display noradrenergic and dopaminergic reuptake inhibition, serotonin $2 \mathrm{C}$, muscarinic and sigma 1 receptor antagonism, as well as inhibition of nitric oxide synthesis and of various cytocrome P450 enzymes. During the first days of treatment there may be adverse events due to rapidly increased serotonin levels in discrete regions of the body. These effects are usually mild and resolve due to desensitization of post-synaptic receptors. However, Wisner et al (11) recommended starting with half the usual SSRI dose in women with PPD due to increased 
initial sensitivity, which resulted in severe headache in several subjects in a randomized trial. Because of their relative safety in overdose, SSRIs are widely prescribed and are often first choice antidepressants. Data published since the Weissman et al's (10) paper are consistent with their findings.

\section{CITALOPRAM}

Four controlled studies and one case report on citalopram have been published since June, 2002. Heikkinen et al. (14) enrolled 11 mothers taking citalopram (20-40 mg daily) during pregnancy and lactation and a control group of 10 drug-free mothers. Outcome measures were infant weight and neurological development through 1 year. The weights of infants of citalopram-treated mothers did not differ from the weights of infant with drug-free mothers. The neurological development of all infants was judged as normal by pediatricians and physiotherapists. The authors monitored plasma and breast milk levels of citalopram and its active metabolites (didesmethylcitalopram and desmethylcitalopram) during pregnancy and for up to 2 months after delivery. The level of the drug in breast milk decreased across time as expected (week $2=445 \pm 141 \mathrm{nmol} / \mathrm{L}$; month $2=278 \pm 91 \mathrm{nmol} / \mathrm{L}$ ). Compared to the level of the drug in the breast milk, the infant serum concentrations were very low (week $2=9.8 \pm 6.5$ $\mathrm{nmol} / \mathrm{L}$; month $2=3.3 \pm 2.2 \mathrm{nmol} / \mathrm{L}$ ). The mean estimated infant citalopram dose was $0.3 \%$ at 2 weeks and $0.2 \%$ at 2 months after delivery. In line with the Weissman et al. (10) findings, the milk to plasma ratios (M/P) ranged from 1.2 to 3.3 for citalopram, from 1.3 to 4.1 for desmethylcitalopram and from 1.1 to 4.6 for didesmethylcitalopram. Interestingly, the $\mathrm{M} / \mathrm{P}$ of all three compounds decreased significantly during the first two months after birth. These results suggested minimal exposure of the infant to citalopram and no evidence of clinical impact at 1 year of age.

Hendrick et al (15) compared 78 nursing infants with mothers taking a variety of SSRIs and 6 month old breastfed infants from normative population. The outcome was weight at 6 months of age, and no difference was found between groups. Only 3 infants were exposed to citalopram and no data on infant exposure level was provided. Berle et al (16) compared 26 breastfed infants whose mothers were treated with a variety of SSRIs in monotherapy (mean age 19 weeks) with 68 nursing infants whose mothers were drug-free (mean age 16 weeks). Ten infants (mean age 18 weeks) were in the citalopram-treated group. The short-term adverse events did not differ between the two groups. The authors found detactable but low serum levels of citalopram in 6 of the 10 infants (mean level $1.9 \mathrm{mmol} / \mathrm{L}$ ). The authors also measured the cytochrome P450 genotypes of CYP2C19 to identify poor metabolizers who are likely to develop drug-specific adverse events. One citalopram-treated mother was identified as a poor metabolizer (homozygous for the inactivating CYP2C19*2 mutation). Although this mother had the highest serum drug concentration $(394 \mathrm{nmol} / \mathrm{L})$ among the citalopram-treated group, her twin infants, extensive metabolizers (both heterozygous for the same mutation) had low serum levels of citalopram ( 2 and $3 \mathrm{mmol} / \mathrm{L}$ respectively) (Berle JO, personal communication:January 29, 2009).

Lee et al (17) compared the frequency of signs in 31 infants with depressed citalopram-treated mothers, 12 infants with depressed mothers treated with a variety of SSRIs, and 31 infants with healthy drug-free mothers. There were no significant differences between the groups in the frequency of infant signs as reported by the mothers and physicians. Among the infants of citalopram-treated mothers, $9.7 \%$ had decreased feeding, colic, and irritability compared to no signs in infants with mothers treated with other SSRIs, and any intervention was required. No measures of infant exposure were provided. Franssen et al. (18) reported a case of an infant of a mother taking citalopram (40 mg daily) during pregnancy and breastfeeding. The infant had irregular breathing, hypotonia, sleep disruption through 3 weeks after delivery that gradually resolved Consistent with Heikkenen et al (14), the relative dose at 25 days and 53 days after 
delivery were respectively $0.9 \%$ and $1.8 \%$, and the $\mathrm{M} / \mathrm{P}$ were 2.0 and 2.8 , respectively, at the same time points.

\section{ESCITALOPRAM}

Escitalopram is the therapeutically active S-stereoisomer (enantiomer) of the SSRI citalopram and is noted for its highly selective serotonin reuptake inhibition. Since June 2002, one study with small sample and four case reports have been published. Rampono et al (19) evaluated the exposure to escitalopram and its active metabolite in 8 infants of mother taking a median dose of $10 \mathrm{mg}$ daily of the drug. The $\mathrm{M} / \mathrm{P}$ and the relative dose of escitalopram plus demethylescitalopram were 2.2 and 5.3\% respectively. Neither short-term adverse events nor developmental abnormalities were noted by a neonatologist. Gentile (20) reported a case of a depressed mother who took escitalopram ( $20 \mathrm{mg}$ daily) at week 24 of gestation and discontinued the therapy gradually over the 2 month period before the delivery. The mother restarted escitalopram at the same dosage post-birth. The infant had no adverse events, as evaluated by a pediatrician through 3 months of age, but no measures of infant exposure were provided.

Potts et al 1(21) reported an case of necrotizing enterocolities (NEC) on the fifth postnatal day in a term neonate exposed to escitalopram ( $20 \mathrm{mg}$ daily) in utero and during breastfeeding. The mother continued taking escitalopram and discontinued breastfeeding. The authors commented on the possible mechanisms that may increase the risk of NEC, including a hypercoagulable state resulting from SSRI withdrawal, as well as vasoconstriction due to the inhibition of nitric oxide production by SSRIs. It is important to note that causality cannot be inferred from a single case report; however, cases of NEC have been previously reported in 2 term neonates whose mothers took paroxetine during pregnancy in association with other agents (desipramine and buspirone respectively)(22). The interesting aspect of the cases above is that NEC is unusual in full term infants.

In a case of a mother taking escitalopram ( $20 \mathrm{mg}$ daily) in combination with reboxetine ( $4 \mathrm{mg}$ daily), the relative dose of escitalopram was $4.6 \%$ and no adverse events were observed in the nursing infant (23). Castberg et al (24) evaluated exposure levels and short term adverse events in a nursing infant with escitalopram-treated mother at 1 week and 7.5 weeks of age. At 1 week, the mother was taking escitalopram ( $5 \mathrm{mg}$ daily) and at week 7.5 increased the dose to $10 \mathrm{mg}$ daily and added valproate $(1200 \mathrm{mg} /$ day). The relative doses of escitalopram at 1 and 7 weeks after delivery were $5.1 \%$ and $7.7 \%$, respectively, and $\mathrm{M} / \mathrm{P}$ were 2.49 and 2.03 at the same time points. The infant did not have clinical abnormalities as reported by the mother and the general practitioner.

\section{FLUOXETINE}

The SSRI fluoxetine is characterized by its long half life, which ranges from 1 to 3 days after a single dose and from 4 to 6 days after a long term use. Similarly, its active metabolite. norfluoxetine has a half-life as long as 16 days. Its pharmacokinetic characteristics explains why fluoxetine is more likely than other SSRIs to result in quantifiable levels in breast milk and infant serum. Additionally, in utero exposure may result in prenatal loading which contributes to post-birth infant serum levels.

Since 2002, five controlled and two uncontrolled studies have been published. In Heikkinen et al (25) 11 mothers taking fluoxetine (20-40 mg daily) during pregnancy and lactation and a control group of 10 medication-free mothers were recruited. Their infants were compared on weight and neurological development through 1 year. Infant weights were not significantly different between the two groups and the neurological development of all infants was judged as normal by pediatricians and physiotherapists. The authors monitored plasma and breast 
levels of fluoxetine and its active metabolite norfluoxetine during pregnancy through to 2 months after delivery with a single breast milk and infant plasma sample at several time points. The infant plasma concentration of fluoxetine plus norfluoxetine decreased during the first two months (ranging from $334 \pm 173 \mathrm{nmol} / \mathrm{L}$ the second day to $22 \pm 14$ at 2 months after delivery). The M/P ranged from 0.3 to 2.2 and from 0.1 to 1.7 , respectively, for fluoxetine and norfluoxetine. The relative dose of fluoxetine plus norfluoxetine was $2.4 \%$ at 2 weeks and 3.8 $\%$ at 2 months after delivery. These results suggested minimal exposure of the infant to the drug. No evidence of short term adverse events or neurodevelopmental impact at 1 year of age was observed. In two controlled studies considering a variety of SSRIs, two lactating women were fluoxetine-treated. Berle et al (16) detected only the active metabolite norfluoxetine in the serum $(47 \mathrm{nmol} / \mathrm{L})$ of one nursing infant, and no adverse events were reported. In the single case of breastfed infant of a fluoxetine-treated mother described by Lee et al (17) any adverse effects was noted. In another study 29 breastfed infants did not differ in weight at 6 months compared to a normative population (15). In both studies any measure of infant exposure was provided. Oberlander et al (26) found detectable serum levels of fluoxetine in 7 infants with prenatal and postnatal exposure to fluoxetine (mean dose of $21.4 \mathrm{mg}$ daily). Fluoxetine was detectable in 2 of the 7 infants serum (mean level $2 \mathrm{ng} / \mathrm{mL}$ ), and the M/P was 0.65 . Kim et al (27) evaluated 23 women taking fluoxetine (10-30 mg daily) and collected samples at a mean time point of 3.7 months postpartum. The relative dose of fluoxetine and norfluoxetine were 0.54 and 0.57 respectively, and the M/P for the two compounds were 0.62 and 0.60 , respectively.

Epperson et al (28) studied the effect of serotonin uptake in platelets in 11 mother-infant pairs (infant mean age at the start of the study before and after 4 to 12 weeks of maternal treatment with fluoxetine (20-40 mg daily). While the mothers showed the expected marked decline in platelet serotonin, only one infant had little or no decline in platelet serotonin. Moreover, no adverse events were noted in the nursing infants. Because platelets and neurons share the same serotonin transporter, this report suggested that fluoxetine delivered to the infant through breast milk had minimal impact on serotonin blockade in the infant brain.

\section{FLUVOXAMINE}

Fluvoxamine has no active metabolites and its mean half-life is 15.6 hours, the shortest halflife among all SSRIs. Since Weissman et al's paper (10), two controlled studies (with fluvoxamine one among a variety of SSRIs) and one case report have been published. Hendrick et al (15) reported that 3 infants exposed to fluvoxamine did not differ in weight compared to unexposed breast fed infants at 6 months. Lee et al (17) reported a single case of nursing infant with fluvoxamine-treated mother, with any noted adverse event. In both studies no measures of infant exposure was provided. Gentile et al (29) reported a case of infant breastfed partially for 3 months. The mother took fluovoxamine ( $200 \mathrm{mg}$ daily) and quetiapine ( $400 \mathrm{mg}$ daily). No clinical abnormalities were noted; however, the mother discontinued breastfeeding due to insufficient milk production. No measures of infant exposure were provided.

\section{PAROXETINE}

Since June 2002, four controlled studies (two of those considering paroxetine among a variety of SSRIs) and four cases have been published. Berle et al (16) calculated infant exposure to paroxetine in 6 breastfed infants of mean age of 16 weeks. Paroxetine was not detectable in any infant serum. No evidence of short-term adverse events was reported. The authors also measured the Cytochrome P450 genotypes of CYP2D6 to identify poor metabolizers who are likely to develop drug-specific adverse events. One mother and her infant were identified as carriers of the CYP2D6 poor metabolizer profile (both homozygous for the inactivating CYP2D6*4 mutation). Despite the mother having the highest serum drug concentration among the paroxetine-treated mothers $(210 \mathrm{nmol} / \mathrm{L})$, paroxetine was non detectable in the serum of 
her infant who was also poor metabolizer. In Hendrick et al (15) 15 nursing infants with mother taking fluoxetine did not differ in weight compared to unexposed breast fed infants at 6 months (15). In Lee et al (17) 3 nursing infants with mothers paroxetine-treated did not have any adverse effect. In both studies any measure of infant exposure was provided. Merlob et al (30) compared weight gain and neurodevelopment at 3, 6, 12 months of age in 27 nursing infants with paroxetine-treated mothers, 27 nursing infants with drug-free mothers, and 19 bottle-fed infants of drug-free mothers. The weight at 6 and 12 months did not differ between the groups and development was normal in all infants. One infant with paroxetine-treated mother developed irritability. No measures of infant exposure were provided. Misri et al (31) reported 4 cases of breastfed infants of mothers treated with paroxetine in association with quetiapine. In only one infant was the breast milk level of paroxetine detectable $(776 \mathrm{nmol} / \mathrm{L})$ and the estimated level of paroxetine exposure were less than $0.26 \mathrm{mg} / \mathrm{kg}$ per day. Two infants showed typical development, whereas two infants showed mild delay but they had no detectable levels of the drug in the milk.

\section{SERTRALINE}

Since June 2002, four controlled studies (three of those considering sertraline among a variety of SSRIs), one non-controlled study and one case report have been published. In Lee et al (17), only two cases were sertraline-treated and no adverse effects were noted (data on infant exposure are not shown). In Hendrick et al., (15) 25 breastfed infants of sertraline-treated mothers did not differ in weight from control infants at 6 months. No measure of infant exposure was provided. Oberlander et al (26) found detectable but low serum levels of sertraline in 4 infants at 2 months of age in mothers who had prenatal and postnatal treatment (mean dose 87 $\mathrm{mg}$ daily). Sertraline was not detectable in any of the infants, and the M/P was 1.02. Stowe et al (32) evaluated exposure to sertraline and its active metabolite desmethylsertraline in 26 breastfed infants whose mothers were a mean dose of $124 \mathrm{mg}$ daily. Sertraline and its metabolite were detectable in $18 \%$ and $50 \%$ of the cases respectively. Using a mathematical model, the authors found the relative dose of sertraline and its metabolite was 0.54 of the maternal dose. No adverse effects were reported in the infants. Since the highest concentrations of the compounds were found in the hindmilk 8-9 hours after ingestion, the authors suggested that reduction of infant exposure could be achieved by discarding breast milk at 8 to 9 hours after maternal drug intake. This strategy also has been recommended by the Academy of Breastfeeding Medicine (33). However, whether this strategy produces a clinically meaningful reduction in an already low exposure remains theoretical.

In Wisner et al. (11) 13 women in the SERT group were breastfeeding and provided motherinfant samples. The mean age of the nursing infants was 5.9 weeks. Sertraline was nondetectable (ND) in the infant serum $(<2 \mathrm{ng} / \mathrm{mL})$ and the metabolite N-desmethyl-SERT ranged from ND to $6 \mathrm{ng} / \mathrm{mL}$. No adverse events was noted. In a case report by Hackett et al (23) sertraline (300 $\mathrm{mg}$ daily) was taken in combination with reboxetine (8 $\mathrm{mg}$ daily). The relative dose of sertraline plus its active metabolite (demethylsertraline) was 3.7\%. No shortterm adverse events or neurodevelopment abnormalities according to the Denver development assessment were noted.

\section{SEROTONIN AND NORADRENALINE-REUPTAKE INHIBITORS (SNRI)}

SNRIs (venlafaxine, desmeethylvenlafaxine, and duloxetine) display their therapeutic effects through the combined inhibition of the reuptake of serotonin and noradrenaline, and less potent inhibition of dopaminergic reuptake. The venlafaxine metabolite (desmthylvenlafaxine) has been recently released, but no data are available. 


\section{VENLAFAXINE}

Since June 2002, one controlled study (considering venlafaxine among a variety of SSRIs) and two cases have been published. In Berle et al. (16) only 3 cases were venlafaxine-treated. Venlafaxine was detected in only one infant, whereas its metabolite O-desmethylvenlafaxine was detected in all infats. The infant serum concentration of venlafaxine plus its metabolite $\mathrm{O}$ desmethylvenlafaxine was $91 \mathrm{nmol} / \mathrm{L}$, and the relative dose was 10.2 (mean values). In Misri et al (31). 2 nursing infants of venlafaxine-treated mothers taking $75 \mathrm{mg}$ and $225 \mathrm{mg}$ daily, respectively, showed detectable levels of the drug in the breast milk ( $371 \mathrm{nmol} / \mathrm{L}$ and 1179 $\mathrm{nmol} / \mathrm{L}$ respectively) and no developmental abnormalities at one year of age. Venlafaxine delivered through breast milk has been proposed as a strategy to attenuate withdrawal syndrome after in utero exposure (34).

\section{DULOXETINE}

Duloxetine is indicated not only for the treatment of depression and anxiety disorder but also for the treatment of neuropathic pain associated with diabetes. Duloxetine has an elimination half-life of about 12 hours (range 8 to 17 hours) and its major circulating metabolites do not contribute significantly to the pharmacologic activity.

Since June 2002, only one uncontrolled study has been published. Lobo et al (35) evaluated the pharmacokinetics of the drug in 6 women taking $40 \mathrm{mg}$ of duloxetine every 12 hours for 3.5 days. After the seventh dose plasma and milk samples were obtained over 12 hours. The milk to $\mathrm{M} / \mathrm{P}$ was 0.25 , and the relative dose of duloxetine was $0.14 \%$. Infant adverse events were not evaluated.

\section{NORADRENERGIC AND SPECIFIC SEROTONERGIC ANTIDEPRESSANT (NaSSA) \\ MIRTAZAPINE}

Mirtazepine's therapeutic mechanism is by blocking presynaptic alpha- 2 adrenergic receptors that normally inhibit the release of the noradrenaline and serotonin. Its half life is 37 hours. Since June 2002, one uncontrolled study and two case reports have been published and represent the first studies providing quantitative data on the infant exposure to NaSSA antidepressants. Kristensen et al. (36) calculated the transfer of mirtazapine and its metabolite into breast milk in 8 infants with mirtazapine-treated mothers (median dose $38 \mathrm{mg}$ daily). The mean $\mathrm{M} / \mathrm{P}$ for mirtazapine and desmethylmirtazapine were 1.1 and 0.6 , and the relative dose of the two compounds combined was $1.9 \%$. Aichhorn et al (37) described a case of a nursing infant with mother taking $30 \mathrm{mg}$ daily of mirtazapine. The drug was present in the breast milk after 6 weeks of treatment ( 7 and $18 \mathrm{ng} / \mathrm{ml}$ in the foremilk and hindmilk, respectively), and no psychomotor abnormalities were noted by a pediatrician. In the case reported by Klier et al. (38), although mirtazapine was detectable in the breast milk 10 hours after the mother's intake of $22.5 \mathrm{mg}$ of the drug $(61 \mathrm{ng} / \mathrm{ml}$ in the foremilk, $90 \mathrm{ng} / \mathrm{ml}$ in the hindmilk), the infant serum levels were undetectable at 12.5 hours postdose.

\section{NOREPINEPHRINE AND DOPAMINE REUPTAKE INHIBITOR (NDRI) BUPROPION}

Bupropion acts as a norepinephrine and dopamine reuptake inhibitor, and nicotinic antagonist. Its half life is 20 hours and it is converted into multiple active metabolites, the most potent of which is 6-hydroxy-bupropion. Bupropion is often used as an augmentation strategy to SSRIs or SNRIs. Bupropion is not associated with weight gain or sexual dysfunction. 
Since June 2002, five single cases and one cohort study have been published. Baab et al (39) calculated infant exposure to buproprion and its active metabolite in 2 nursing infants of mothers taking $150 \mathrm{mg}$ of bupropion. Serum infant levels were undetectable in both cases $(<5$ and $<10 \mathrm{ng} / \mathrm{mL}$ ) at respectively 17 weeks and 39.5 weeks of age. The pediatricians did not observe any adverse events (39). Chaudron et al (40) reported a single case of seizure in a 6 months nursing infant after four days of the mother's treatment with sustained release bupropion (starting dose $150 \mathrm{mg}$ and increase to $300 \mathrm{mg}$ after 36 hours). The mother discontinued buproprion and started sertraline, with no further seizures in the infant. No laboratory confirmation of either maternal or infant exposure were obtained. In a open trial of sustained release bupropion for PPD (41), 2 women were breastfeeding and reported no adverse events in their infants. No additional data on infant exposure were provided. In the study by Haas et al (42) 10 healthy postpartum volunteers took $150 \mathrm{mg}$ bupropion for 3 days and then $300 \mathrm{mg}$ for 4 days. Bupropion and its active metabolites (hydroxybupropion, erythrohydrobupropion, threohydrobupropion) were present in breastmilk (mean levels 45.2, 104.6, 72.1, $459 \mathrm{ng} / \mathrm{mL}$, respectively). The average M/P for bupropion, hydroxybupropion, erythrohydrobupropion, and threohydrobupropion were $2.8,0.1,0.9$, and 1.2 , respectively. The average infant exposure was expected to be $2 \%$ of the standard maternal dose on a molar basis. No adverse effects were noted by the mothers.

\section{PRACTICAL RECOMMENDATIONS}

Recommendations are based on single case reports, studies with small samples (rarely casecontrol), consensus and expert opinions (Level B,C,D of recommendation according to the UK National Health Service)(43). Moreover, the empirical studies with the largest samples ( $\geq$ 20) included a variety of antidepressants which limits data on specific agents. Practical recommendations derived from published data provide clinical guidance as scientific evidence evolves.

To date three scientific commissions developed practical guidelines regarding the use of antidepressants during breastfeeding (33)(44)(45). They converge in recommending as follows: 1) The choice of the specific treatment of breastfeeding mothers should derive from a personalized risk-benefit analysis considering the risk of untreated maternal illness, the risk/ benefit of the specific treatment, the risk/benefit of breastfeeding for the mother/infant pair (46) (see Figure 1). 2) Complete information regarding availability and risk/benefit of the treatments should be provided in order to facilitate the patient's informed decision making. 3) Non-pharmacological interventions, such as psychotherapy (6) should be considered first line for the treatment of mild to moderate postpartum depression. 4) Antidepressants (alone or in combination with non-pharmacological interventions) should be considered for women with moderate to severe depression or not responding (or declining) psychological treatments. 5) The choice of the specific antidepressant should be based on clinical factors, particularly previous efficacious treatments. 6) If the woman is suffering from the first lifetime episode, sertraline or paroxetine should be the agents of first choice. 7) The antidepressant should be started at the lowest effective dose and increased slowly. 8) Monotherapy is preferred. 9) Mothers and infants' clinical conditions should be monitored, particularly for sick or low birth weight infants. 10) Serum infant levels are not recommended routinely but only if clinically indicated and by a research laboratory.

Controversy exists as to whether the current assessments are accurate in estimating the infant exposure to drugs through breastfeeding, and innovative laboratory methods to reflect infant serum concentration using breast milk have been explored (47)(48). However, to date the available biological parameters lack reliability and are not correlated with clinical outcomes. Gentile et al (49) proposed a Specific Safety Index (BI-ASI) based on the frequency and degree of severity of adverse events in nursing infants. Similarly, based on the available scientific 
literature, Hale (50) developed Lactation Risk Categories for a variety of medications, including antidepressants, that can be consulted in clinical practice.

\section{CONCLUSIONS}

For this article we have updated the material of the paper published by Weisman et al. in 2004 (10), expanding the data for newer agents (Table n.1). In line with their results recent findings confirm that antidepressants differ with respect to infant exposure. Data remain limited, correlations with clinical outcome require increased methodological sophistication, and rates of adverse events have not been established. We cannot conclude that undetectable levels of drug have no short-or long- term effects, nor that measurable drug levels in infant serum are associated with adverse events. Moreover, the recent studies evaluating the long-term effects of antidepressants delivered through breast milk are limited to infants one year of age and are often confounded by prenatal exposure to either drug or major depression. The only indirect biological evidence that serotonin transmitters may not be affected are the studies of Epperson et al (28)(51) with no observed platelet serotonin effects for breastfed infants with mothers treated with sertraline or fluoxetine.

Because there are limited data on preterm infants, theoretical risks based on pharmacological principles (52) are invoked (i.e incomplete nephrogenesis, and undeveloped metabolizing enzymes). In Wisner et al. (53) one preterm infant (36 weeks) of NTP-treated mother presented with drug and metabolite levels similar to those of full term infants whose mothers began taking NTP at birth. Since short- and long- term benefits of breast milk apply especially for preterm infants, collection of data on those women who provide breast milk to preterm infants would be a notable research contribution. This work would require careful attention to the amount of breast milk compared to supplemental formula that the infant receives, as well as other medications, both given to the mother pre-partum and to the infant.

We return to the questions listed in the introduction of this manuscript. 1) The exposure of infants to relatively low doses of antidepressants through breast milk must be juxtaposed with that of untreated maternal PPD, which has well-established negative consequences in multiple domains (7)(8)(54) which is reviewed in this supplement (Brand and Brennan), if the woman's treatment of choice is drug therapy. For example, in a recent study (15) infants whose mothers experienced chronic postpartum depression had significantly lower weights compared to infants whose mothers had short episodes of depression and infants of mother who did not have depression. In the same study the exposure to antidepressants during breastfeeding did not affect infant's weight. 2) Breastfeeding and treatment with antidepressant medications are not mutually exclusive. The benefits of breastfeeding for maternal and infants health are welldocumented (1)(2).3). Sertraline and paroxetine (among SSRIs) and nortriptyline and imipramine (among TCAs) are the most evidence-based medications for use during breastfeeding because of similar findings across multiple laboratories, usually undetectable infant serum levels and no reports of short term adverse events. 4) Evidence on the more recently released antidepressants are represented by uncontrolled studies and case reports and are limited to short-term evaluation. These agents should not be first choices in postpartum women who want to continue breastfeeding unless demonstrated efficacy in past episodes is clear and no new contraindications exist for their use in the individual woman. 5) Although strategies to reduce infant exposure to antidepressants have been suggested (i.e. discarding the breast milk obtained during the peak serum level), whether such strategies produce a clinically meaningful reduction in an already low exposure has not been established. 6) Long-term follow up of infants exposed through breastfeeding, with control for maternal depression, remains an understudied area. However, exposure of the fetus during pregnancy is substantially greater than that during lactation, and the minimal data available on longer term development has not demonstrated delays (55)(56)(57). 


\section{Acknowledgments}

Dr. Wisner has received grant support from Pfizer in the past and is currently on the speaker's bureau of Glaxo-SmithKline. Dr. Wisner's effort was supported primarily by National Institute of Mental Health R01 MH60335; also by National Institute of Mental Health R01 MH071825 and R01 MH075921. She receives research support from the State of Pennsylvania and the Heinz Foundation.

The authors thank Dr. Jan Oeystein Berle for sharing unpublished data.

\section{References}

1. Goldman AS, Hopkinson JM, Rassin DK. Benefits and risks of breastfeeding. Advances in Pediatrics 2007;25:275-304. [PubMed: 17918475]

2. American Academy of Pediatrics Policy statement: breastfeeding and the use of human milk. Pediatrics 2005;115:496-506. [PubMed: 15687461]

3. World Health Organization. Infant and Young Child Feeding. Geneva, Switzerland: World Health Organization; 2003 [Jan 26, 2009]. Available at http://www.who.int/en/

4. Gaynes B, Gavin N, Meltzer-Brody S, et al. Perinatal depression: prevalence, screening accuracy, and screening outcomes. Evid Rep Technol Assess (Summ) 2005;119:1-8. [PubMed: 15760246]

5. Pearlstein TB, Zlotnick C, Battle CL, et al. Patient choice of treatment for postpartum depression: a pilot study. Arch Womens Ment Health 2006;9:303-308. [PubMed: 16932988]

6. O'Hara MW, Stuart S, Gorman LL, et al. Efficacy of interpersonal psychotherapy for postpartum depression. Arch Gen Psychiatry 2000;57:1039-1045. [PubMed: 11074869]

7. Pilowsky DJ, Wickramaratne PJ, Rush AJ, et al. Children of currently depressed mothers: a STAR*D ancillary study. J Clin Psychiatry 2006;67:126-36. [PubMed: 16426099]

8. Logsdon CM, Wisner KL, Pinto-Foltz MD. The impact of postpartum depression on mothering. JOGNN 2006;35:652-658. [PubMed: 16958723]

9. American Psychiatric Association Practice Guidelines. [Jan 27, 2009]. Available at http://www.psych.org

10. Weissman AM, Levy BT, Hartz AJ, et al. Pooled analysis of antidepressant levels in lactating mothers, breast Milk, and nursing infants. Am J Psychiatry 2004;161:1066-1078. [PubMed: 15169695]

11. Wisner KL, Hanusa BH, Perel JM, et al. Postpartum depression. A aandomized trial of sertraline versus nortriptyline. J Clin Psychopharmacol 2006;26:353-360. [PubMed: 16855451]

12. Eberhard-Gran M, Eskild A, Opjordsmoen S. Use of psychotropic medications in treating mood disorders during lactation. Practical recommendations. CNS Drugs 2006;20:187-198. [PubMed: 16529525]

13. Whitby DH, Kelly M, Smith KM. The use of tricyclic antidepressants and selective serotonin reuptake inhibitors in women who are breastfeeding. Pharmacotherapy 2005;25:411-425. [PubMed: 15843288]

14. Heikkinen T, Ekblad U, Kero P, et al. Citalopram in pregnancy and lactation. Clin Pharmacol Ther 2002;72:184-91. [PubMed: 12189365]

15. Hendrick V, Smith LM, Hwang S, et al. Weigh gain in breastfed infants of mother taking antidepressant medications. J Clin Psychiatry 2003;64:410-412. [PubMed: 12716242]

16. Berle JO, Steen VM, Aamo TO, et al. Breastfeeding during maternal antidepressant treatment with serotonin reuptake inhibitors: infant exposure, clinical symptoms, and cytochrome P450 genotypes. J Clin Psychiatry 2004;65:1228-1234. [PubMed: 15367050]

17. Lee A, Woo J, Ito S. Frequency of infant adverse events that are associated with citalopram use during breast-feeding. Am J Obstet Gynecol 2004;190:218-21. [PubMed: 14749663]

18. Franssen EJF, Meijs V, Ettaher F, et al. Citalopram serum and milk levels in mother and infant during lactation. Ther Drug Monit 2006;28:2-4. [PubMed: 16418683]

19. Rampono J, Hackett $\mathrm{P}$, Kristensen JH, et al. Transfer of escitalopram and its metabolite demethylescitalopram into breastmilk. Br J Clin Pharmaco 2006;62:316-322.

20. Gentile S. Escitalopram late in pregnancy and while breastfeeding. Ann Pharmacother 2006;40:1696. [PubMed: 16912243] 
21. Potts AL, Young KL, Carter BS, et al. Necrotizing enterocolitis associated with in utero and breast milk exposure to the selective serotonin reuptake inhibitor, escitalopram. J Perinatol 2007;27:120122. [PubMed: 17262045]

22. Stiskal JA, Kulin N, Koren G, et al. Neonatal paroxetine withdrawal syndrome. Arch Dis Child Fetal Neonatal Ed 2001;84:F134-F135. [PubMed: 11207233]

23. Hackett LP, Ilett KF, Rampono J, et al. Transfer of reboxetine into breastmilk, its plasma concentrations and lack of adverse effects in the infant. Eur J Clin Pharmacol 2006;62:633-638. [PubMed: 16699799]

24. Castberg I, Spigset O. Excretion of escitalopram in breast milk. J Clin Psychopharmacol 2006;26:536537. [PubMed: 16974204]

25. Heikkinen T, Ekblad U, Palo P, et al. Pharmacokinetics of fluoxetine and norfluoxetine in pregnancy and lactation. Clin Pharmacol Ther 2003;73:330-337. [PubMed: 12709723]

26. Oberlander, Grunau RE, Fitzgerald C, et al. Exposure pain reactivity in 2-month-old infants after prenatal and postnatal selective serotonin reuptake inhibitor medication. Pediatrics 2005;115:411425. [PubMed: 15687451]

27. Kim J, Riggs KW, Misri S, et al. Stereoselective disposition of fluoxetine and norfluoxetine during pregnancy and breast-feeding. Br J Clin Pharmacol 2005;61(2):155-163. [PubMed: 16433870]

28. Epperson CN, Jatlow PI, Czarkowski K, et al. Maternal fluoxetine treatment in the postpartum period: effects on platelet serotonin and plasma drug levels in breastfeeding mother-infant pairs. Pediatrics 2003;112:425-429.

29. Gentile S. Quetiapine-fluvoxamine combination during pregnancy and while breastfeeding. Arch Womens Ment Health 2006;9:158-159. [PubMed: 16683078]

30. Merlob P, Stahl B, Sulkes J. Paroxetine during breast-feeding: infant weight gain and maternal adherence to counsel. Eur J Pediatr 2004;163:135-139. [PubMed: 14745552]

31. Misri S, Corral MMD, Wardrop AA, et al. Quetiapine augmentation in lactation. A series of case reports. J Clin Psychopharm 2006;26:508-511.

32. Stowe ZN, Hostetter AL, Owens MJ, et al. The pharnachokinetic of sertraline excretion into human breast milk: determinants of infant serum concentrations. J Clin Psychiatry 2003;64:73-80. [PubMed: 12590627]

33. ABM Clinical Protocol \#18: Use of antidepressants in nursing mothers. The Academy of Breastfeeding Medicine Protocol Committee. Breastfeed Med 2008;3:44-52. [PubMed: 18333769]

34. Koren G, Moretti M, Kapur BJ. Can venlafaxine in breast milk attenuate the norepinephrine and serotonin reuptake neonatal withdrawal syndrome? Obstet Gynaecol Can 2006;28:299-302.

35. Lobo ED, Loghin C, Knadler MP, et al. Pharmacokinetics of duloxetine in breast milk and plasma of healthy postpartum women. Clin Pharmacokinet 2008;47:103-109. [PubMed: 18193916]

36. Kristensen JH, Ilett KF, Rampono J, et al. Transfer of the antidepressant mirtazapine into breast milk. British Journal of Clinical Pharmacology. Br J Clin Pharmacol 2006;63:322-32. [PubMed: 16970569]

37. Aichhorn W, Whitworth AB, Weiss U, et al. Mirtazapine and breastfeeding. Am J Psychiatry 2004;161:2325. [PubMed: 15569912]

38. Klier CM, Mossahebm N, Lee A, et al. Mirtazapine and breastfeeding: maternal and infant plasma levels. Am J Psychiatry 2007;164:348-349. [PubMed: 17267804]

39. Baab SW, Peindl KS, Piontek CM, et al. Serum bupropion levels in breastfeeding mother-infant pairs. J Clin Psychiatry 2002;63:910-911. [PubMed: 12416600]

40. Chaudron LH, Schoenecker CJ. Bupropion and breastfeeding: a case of a possible infant seizure. J Clin Psychiatry 2004;65:881-882. [PubMed: 15291673]

41. Nonacs RM, Soares CN, Viguera AC, et al. Bupropion SR for the treatment of postpartum depression: a pilot study. Int J Neuropsychopharmacol 2005;8:445-449. [PubMed: 15817137]

42. Haas JS, Kaplan CP, Barenboim D, et al. Bupropion in breast milk: an exposure assessment for potential treatment to prevent post-partum tobacco use. Tob Control 2004;13:52-56. [PubMed: 14985597]

43. Evidence Report/Technology Assessment. Vol. 119. Rockville,MD: Agency for Healthcare Research and Quality; 2005. p. 1-8. 
44. ACOG Practice Bulletin Clinical Management Guidelines for Obstetrician-Gynecologists. Obstet Gynecol 2008;111:1001-1020. [PubMed: 18378767]

45. National Institute for Clinical Excellence: Antenatal and Postnatal Mental Health: Clinical Management and Service Guidance, Clinical Guideline No. 47. London: NICE; 2007.

46. Sit DKY, Wisner KL. Decision making for postpartum depression treatment. Psychiatry Annals 2005;35:577-584.

47. Suri R, Stowe ZN, Hendrick V, et al. Estimates of nursing infant daily dose of fluoxetine through breast milk. Biol Psychiatry 2002;52:446-451. [PubMed: 12242061]

48. Hostetter AL, Stowe ZN, Cox M, et al. A novel system for the determination of antidepressant concentrations in human breast milk. Ther Drug Monit 2004;26:47-52. [PubMed: 14749550]

49. Gentile S. Use of contemporary antidepressants during breastfeeding. A proposal for a specific safety index. Drug Saf 2007;30:107-121. [PubMed: 17253877]

50. Hale, TW. Medications and Mothers's Milk. Thirtheen Edition. Hale Publishing; 2008.

51. Epperson CN, Czarkowski KA, Ward-O'Brien D, et al. Maternal sertraline treatment and serotonin transport in breastfeeding mother-infant pairs. Am J Psychiatry 2001;158:1631-163. [PubMed: 11578995]

52. Alcorn J, McNamara PJ. Ontogeny of hepatic and renal systemic clearance pathways in infants. Part I. Clin Pharmacokinet 2002;41:959-998. [PubMed: 12222995]

53. Wisner KL, Perel JM. Serum nortriptyline levels in nursing mothers and their infants. Am J Psychiatry 1991;148:1234-1236. [PubMed: 1883004]

54. Wisner KL, Chambers C, Sit DKY. Postpartum depression. A major public health problem. JAMA 2006;296:2616-2618. [PubMed: 17148727]

55. Nulman I, Rovet J, Stewart DE, et al. Child development following exposure to tricyclic antidepressants or fluoxetine throughout fetal life: a prospective controlled study. Am J Psychiatry 2002;59:1889-1895. [PubMed: 12411224]

56. Yoshida K, Smith B, Craggs M, et al. Fluoxetine in breastmilk and developmental outcome of breastfed infants. Br J Psychiatry 1998;172:175-178. [PubMed: 9519072]

57. Stowe ZN, Owens MJ, Landry JC, et al. Sertraline and desmethylsertraline in human breast milk and nursing infants. Am J Psychiatry 1997;154:1255-1260. [PubMed: 9286185]

58. Wisner KL, Parry BL, Piontek KM. Postpartum depression. N Engl J Med 2002;347:194-199. [PubMed: 12124409] 


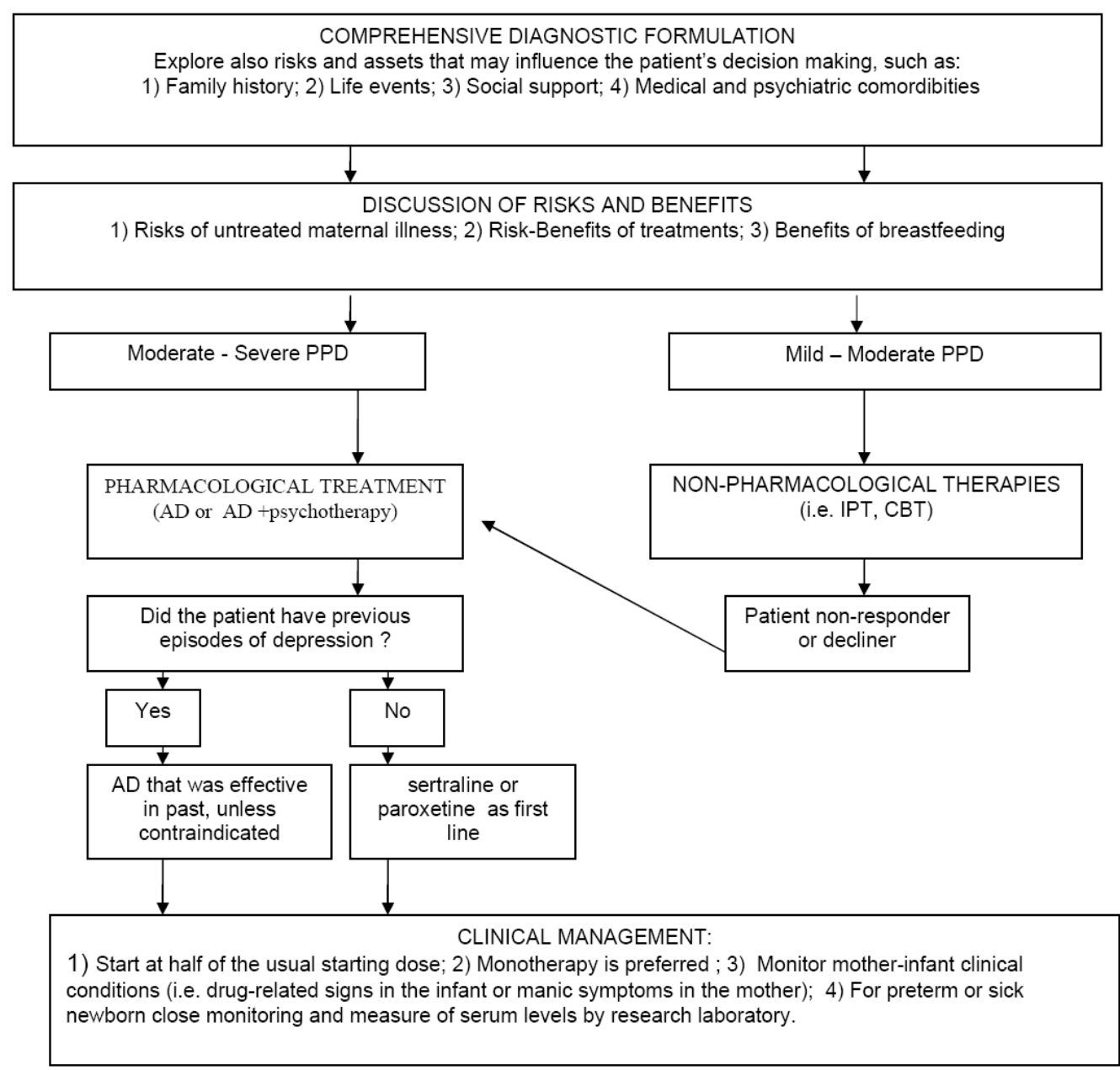

Figure 1. Decision Making for Postpartum Depression Treatment

Source: Adapted from: Wisner et al. (2002) (58), Sit and Wisner (2006) (46)

Abbreviations: $\mathrm{PPD}=$ postpartum depression; $\mathrm{IPT}=$ interpersonal psychotherapy; $\mathrm{CBT}=$ cognitive-behavioral psychotherapy; $\mathrm{AD}=$ antidepressant 
Table 1

Empirical studies published since June, 2002

\begin{tabular}{|c|c|c|c|c|}
\hline Class & Individual Medication & $\begin{array}{l}\text { No. of Empirical } \\
\text { Studies }\end{array}$ & $\begin{array}{l}\text { No. of Nursing } \\
\text { Infants }\end{array}$ & Summary of short and long-term clinical adverse events \\
\hline TCAs & Nortryptyline & 1 & 16 & None \\
\hline \multirow{6}{*}{ SSRIs } & Citalopram & 4 & 54 & $\begin{array}{l}1 \text { case of transient irregular breathing, hypotonia, sleep disruption } \\
\text { through } 3 \text { weeks after delivery (18) }{ }^{1} \text {. } \\
3 \text { cases of infants colic, decreased feeding, irritability/restlessness } \\
\text { respectively (17) } \\
\text { No intervention was required }\end{array}$ \\
\hline & Escitalopram & 4 & 12 & $\begin{array}{l}1 \text { case of infant necrotizing enterocolitis (NEC) in full term infant } \\
\qquad(21)^{l}\end{array}$ \\
\hline & Fluoxetine & 7 & 83 & None \\
\hline & Fluvoxamine & 3 & 5 & None \\
\hline & Paroxetine & 5 & 55 & $\begin{array}{l}1 \text { case of irritability (30). } \\
2 \text { cases of mild neurodevelopmental delay at one year of age (31) }\end{array}$ \\
\hline & Sertraline & 6 & 69 & None \\
\hline \multirow[b]{2}{*}{ SNRIs } & Venlafaxine & 2 & 5 & None \\
\hline & Duloxetine & 1 & 6 & $\begin{array}{l}\text { NE }(35)^{3} \text { PLEASE CLARIFY NE, OR NA AS NOTED IN THE } \\
\text { LEGEND? }\end{array}$ \\
\hline NaSSA & Mirtazapine & 3 & 10 & None \\
\hline NDRI & Bupropion & 4 & 15 & 1 case of seizure $(40)^{4}$ \\
\hline
\end{tabular}

${ }^{1}$ The infant was exposed to the drugs also in utero

2

2 As assessed by the Bayley Scales of Infant Development, Second Edition

${ }^{3} \mathrm{NA}=$ not evaluated

4 The infant was 6-months and the mother was taking sustained released bupropion (starting dose $150 \mathrm{mg} / \mathrm{d}$ ). Any measure of infant exposure was obtained (THIS SEEMS TO BE AN INCOMPLETE STATEMENT)

scalea 